\title{
Dieta del pez espada Xiphias gladius Linnaeus, 1758 en distintas zonas de pesca frente a Chile central durante el otoño de 2004
}

\author{
Diet of swordfish Xiphias gladius Linnaeus, 1758 from different fishing zones \\ off central-Chile during autumn 2004 \\ Katherine Castillo ${ }^{1}$, Christian M. Ibáñez ${ }^{2}$, Carlos González ${ }^{3}$ y Javier Chong ${ }^{1}$ \\ ${ }^{1}$ Departamento de Ecología Costera, Facultad de Ciencias, Universidad Católica de la Santísima Concepción \\ Casilla 297, Concepción, Chile \\ ${ }^{2}$ Instituto de Ecología y Biodiversidad, Departamento de Ciencias Ecológicas, Facultad de Ciencias, Universidad de Chile \\ Casilla 653, Santiago, Chile \\ ${ }^{3}$ Departamento de Pesquería, Instituto de Investigación Pesquera, Colón 2780 \\ Casilla 350, Talcahuano, Chile \\ christianibez@yahoo.com
}

\begin{abstract}
The habitat of the predator and sample size (stomach number) are some of the most important factors to consider in the study of its diet. To investigate these variations we studied the diet of 51 swordfish Xiphias gladius (range: 173-301 cm) in four fishing areas off central Chile $\left(30^{\circ} \mathrm{S}-40^{\circ} \mathrm{S}\right)$ during autumn-winter of 2004 . Frequency of occurrence, numeric and gravimetric methods were used to describe the diet. The sample size was evaluated using rarefaction curves. Prey richness, diversity, dominance, and evenness were calculated and compared between fishing areas. Xiphias gladius mainly fed on squid (10 species) and secondarily fish (5 species), being jumbo squid Dosidicus gigas the main prey in all the fishing areas. Sample size was not enough to adecuately describe the diet of $X$. gladius, and geographical variation could not be assessed.
\end{abstract}

Key words: Swordfish, Xiphias gladius, stomach contents, feeding, cephalopods, diet variations, Chile

\section{Introducción}

El pez espada Xiphias gladius Linnaeus, 1758 es una especie epipelágica y mesopelágica, que se distribuye geográficamente entre los $45^{\circ} \mathrm{N}$ a los $45^{\circ} \mathrm{S}$, es decir, en todos los mares tropicales, subtropicales y templados, tolerando un amplio rango de temperaturas, por lo cual su abundancia y distribución están asociadas con aguas superficiales $>18^{\circ} \mathrm{C} \mathrm{y}<30^{\circ} \mathrm{C}$ (Palko et al. 1981). En aguas de origen oceánico, frente a Chile, su
Resumen.- El hábitat del depredador y el tamaño muestreal (número de estómagos) son algunos de los factores más importantes a considerar en el estudio de su dieta. Para investigar esto se estudió la dieta en 51 peces espada Xiphias gladius (rango: 173-301 cm) en cuatro zonas de pesca en Chile central $\left(30^{\circ} \mathrm{S}-40^{\circ} \mathrm{S}\right)$ durante el otoño-invierno del año 2004. La frecuencia de ocurrencia, métodos gravimétricos y numéricos fueron usados para describir la dieta. El tamaño muestreal fue evaluado usando curvas de rarefacción. La riqueza de presas, diversidad, dominancia, y equitabilidad fueron calculados y comparados entre áreas de pesca. Xiphias gladius se alimenta principalmente de calamares (10 especies) y secundariamente de peces (5 especies), siendo el calamar Dosidicus gigas la presa principal en todas las áreas de pesca. Los tamaños muestreales no fueron suficientes para describir adecuadamente la dieta de $X$. gladius, y la variación geográfica no pudo ser estimada.

Palabras clave: Xiphias gladius, contenido estomacal, alimentación, cefalópodos, variabilidad dietaria, Chile distribución espacial está relacionada con temperaturas superficiales de $14^{\circ} \mathrm{C}$ a $18^{\circ} \mathrm{C}$ (Yáñez et al. 1996). Con respecto a su distribución vertical, se desplaza en aguas superficiales, pero en algunas áreas descienden a grandes profundidades alcanzando hasta 1000 metros o más (Carey \& Robison 1981, Takahashi et al. 2003). Consecuencia de estas características, en los sistemas pelágicos tropicales, $X$. gladius realiza migraciones hacia aguas frías durante el verano con el fin de alimentarse (Palko et al. 1981). 
Debido a que $X$. gladius es una especie relativamente solitaria (Palko et al. 1981), en aguas oceánicas de Chile central se pueden capturar pocos individuos ( $<20$ en una semana), la mayoría maduros entre 130 y $250 \mathrm{~cm}$ de longitud horquilla (Ibáñez et al. 2004), lo que refleja su baja abundancia en estas aguas y por lo que es más frecuente encontrar tiburones (72\%) con respecto al pez espada (21\%) en las faenas de pesca dedicadas a $X$. gladius (Acuña et al. 2002). Entonces es importante considerar que el número de ejemplares de $X$. gladius posibles de obtener para estudios de alimentación no son muy elevados, y por lo tanto se debe evaluar si los tamaños muestreales son representativos para caracterizar la dieta de esta especie en una zona dada. Con respecto a esto, Markaida \& Sosa-Nishizaki (1998) estimaron, en base a la diversidad de presas acumulada, un tamaño muestreal de 20 a 25 individuos para estudios dietarios. Ibáñez et al. (2004) encontraron diferencias en la dieta de $X$. gladius en tres zonas de pesca frente a Chile con tamaños muestreales de 12, 15 y 21 ejemplares respectivamente, pero no evaluaron si el número de ejemplares fue adecuado para realizar las comparaciones.

Varios autores concuerdan en que la alimentación de esta especie está compuesta principalmente de cefalópodos, peces y crustáceos, ocupando los primeros un mayor porcentaje de la dieta (Palko et al. 1981, Bello 1991, Hernández-García 1995, Smale 1996, Ibáñez et al. 2004). Los estudios de dieta de pez espada frente a Chile son escasos y basados en pocas muestras y se remontan al registro de LaMonte \& Marcy (1941), los cuales hallaron sólo calamares en los estómagos. De Sylva (1962) identificó 24 calamares Dosidicus gigas y el pez Sarda chiliensis en siete hembras en el norte de Chile, y concluyó que el pez espada se alimenta cerca de la superficie. Barbieri et al. (1998) reportaron que los peces Macruronus magellanicus y Trachurus murphyi fueron las presas más importantes en frecuencia de ocurrencia (60\% cada uno) en 55 estómagos de $X$. gladius, seguido de los calamares Dosidicus gigas y Loligo gahi (15\%), y el camarón Heterocarpus reedi (10\%). Yatsu (1995) reportó peces y cefalópodos no identificados en 19 estómagos de $X$. gladius. Recientemente, Ibáñez et al. (2004) encontraron que $X$. gladius es un depredador principalmente teutófago $\mathrm{y}$ secundariamente piscívoro, siendo Dosidicus gigas la principal presa en dos de las tres zonas de pesca estudiadas ( $\mathrm{F}=100$ y 75\%), y Onychoteuthis banksii en una de ellas ( $\mathrm{F}=71 \%$ ). A pesar de que la mayoría de los estudios son solamente descriptivos, algunos autores han encontrado variaciones en la dieta entre localidades geográficas (Stillwell \& Kholer 1985, Hernández-García 1995, Markaida \& Sosa-Nishizaki 1998, Ibáñez et al. 2004) y estaciones del año (Stillwell \& Kholer 1985, Peristeraki et al. 2005). La capacidad de $X$. gladius de recorrer largas distancias, ya que es una especie de gran tamaño (>500 kg) y se alimenta de especies que forman cardúmenes (Markaida \& SosaNishizaki 1998, Ibáñez et al. 2004), podría explicar las variaciones en su dieta tanto local como geográficas. Dependiendo del área geográfica en la que se encuentre el pez espada su espectro trófico podría ser muy amplio (superior a 30 especies), o por el contrario reducido alrededor de 10 especies, y por lo tanto la abundancia y diversidad de las especies presas podrían cambiar entre zonas de pesca frente a Chile central.

El objetivo de este estudio fue describir la dieta de $X$. gladius y evaluar si el tamaño muestreal obtenido es representativo para realizar comparaciones en términos de abundancia y diversidad de presas en diferentes zonas geográficas que constituyen sitios de pesca de esta especie en aguas de Chile central.

\section{Materiales y métodos}

Se examinó el contenido de 51 estómagos de pez espada obtenidos a bordo de dos embarcaciones artesanales dedicadas a la captura del recurso con un total de cinco mareas, entre abril y junio de 2004. Sus capturas se agruparon dependiendo de la latitud y longitud en cuatro zonas de pesca (Fig. 1).

A bordo de las embarcaciones, a cada individuo se le registro la longitud horquilla, desde el extremo de la mandíbula inferior hasta la horquilla (LHMI), en centímetros. Los estómagos fueron extraídos y fijados en formalina al $10 \%$ para su posterior análisis en laboratorio. Los estómagos se disecaron para determinar el número y peso de las presas del contenido estomacal. Posteriormente, las distintas presas fueron identificadas a través de la comparación con material de referencia y claves de literatura (Wolff 1982, Nesis 1987, Leible \& Miranda 1989, Kong \& Meléndez 1991, Falabella et al. 1995). 


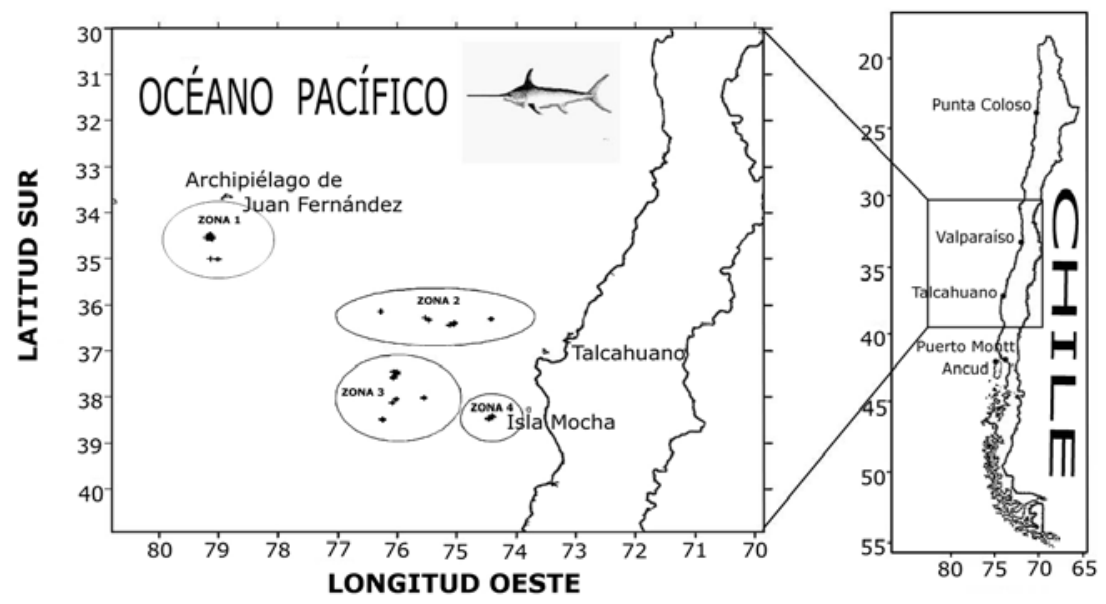

Figura 1

Ubicación geográfica de las zonas de captura de $X$. gladius frente a Chile

Geographic positions of $X$. gladius fishing zones off Chile

Para evaluar si el tamaño muestreal fue representativo, se trazaron curvas de rarefacción (Sanders 1968, Ludwig \& Reynolds 1988) basadas en la abundancia de cada especie presa en los estómagos del pez espada en cada zona de pesca. Esta técnica, desarrollada por Sanders (1968) y corregida posteriormente por Hulbert (1971), calcula el número de especies esperadas en el caso de que todas las muestras poseyesen el mismo número de individuos capturados.

La dieta se describió de acuerdo a los descriptores cuantitativos propuestos por Hyslop (1980): Frecuencia de ocurrencia $(\% \mathrm{~F})$, método numérico $(\% \mathrm{~N})$ y método gravimétrico (\%G).

La comparación de las variaciones de la dieta en relación con las zonas de pesca se realizaron mediante la frecuencia de ocurrencia (F) de cada ítem presa usando una tabla de contingencia de máxima verosimilitud (prueba G) de 4×16 y el estadígrafo de Ji-cuadrado (Zar 1984, Cortés 1997).

Además se calculó la riqueza (número de especies), la diversidad y equitabilidad de Brillouin, y dominancia de Berger-Parker de la dieta para cada ejemplar (Magurran 1988), los que fueron comparados con análisis de varianza (ANOVA) (Zar 1984) entre las zonas de pesca. De la misma forma se comparó la abundancia de presas en cada estómago. Todos los análisis se realizaron en el programa PAST.exe (Hammer et al. 2001).

\section{Resultados}

Los peces espada analizados midieron entre 173 y 301 cm LHMI (promedio de 222 cm; DE 34,7). Todos los ejemplares analizados estaban en el rango de tamaño de madurez sexual (>152 cm, Palko et al. 1981). La longitud de los ejemplares de $X$. gladius capturados no resultó distinta entre las zonas de pesca (ANOVA, F(3, 45) =0,818; $P=0,49$ ).

La falta de tendencia a la asíntota de las curvas de rarefacción indican que el esfuerzo de muestreo no fue suficiente para representar el número de especies presentes en los estómagos de $X$. gladius y que el tamaño muestreal no fue suficiente para describir su alimentación (Fig. 2).

El 96\% de los 51 estómagos de $X$. gladius analizados presentó contenido estomacal. La dieta de $X$. gladius estuvo compuesta principalmente de cefalópodos y en segundo orden de peces. En las cuatro zonas de captura la especie en estudio, $X$. gladius, se alimentó principalmente del calamar Dosidicus gigas, siendo la presa más importante de acuerdo a todos los descriptores utilizados para la dieta. El pez Trachurus murphyi estuvo presente en tres zonas marcando un alto porcentaje en la zona 4 (Tabla 1). 


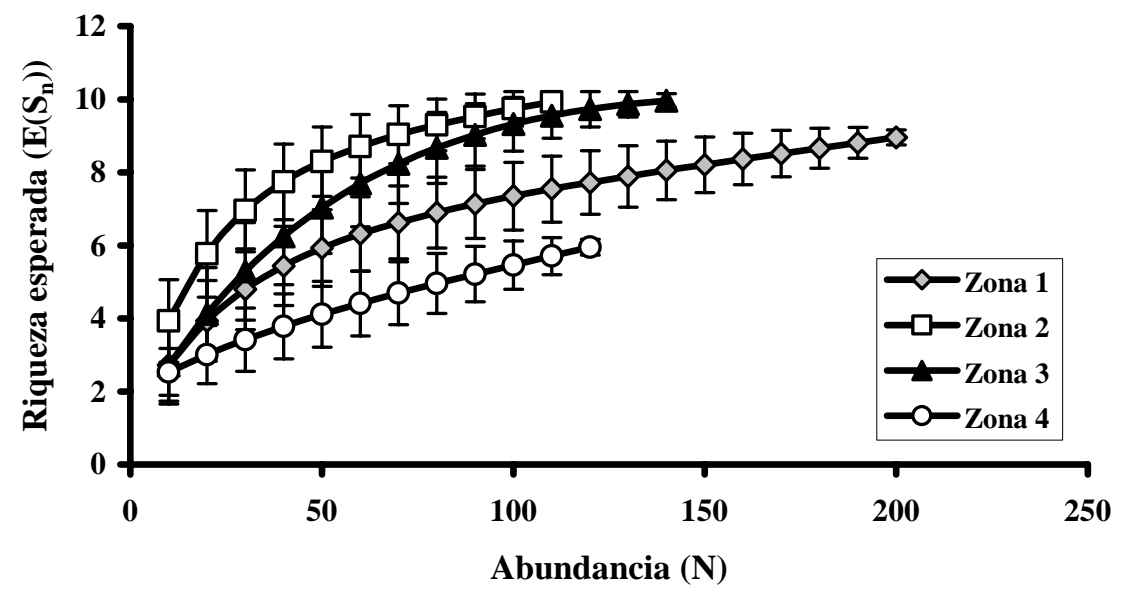

Figura 2

Curvas de rarefacción respecto a la abundancia de las presas en la dieta de $X$. gladius para cada zona de pesca. Las barras representan intervalos de confianza de $95 \%$

Rarefaction curves of prey abundance in the diet of $X$. gladius for each fishing zone. Bars represent 95\% confidence intervals

Tabla 1

Contenido estomacal de $X$. gladius en las cuatro zonas de pesca. Porcentajes de los métodos de frecuencia (F\%), numérico (N\%) y gravimétrico (P\%) de cada ítem dietario

Stomach content of $X$. gladius from four fishing zones. Percent of frequency of occurrence $(\% \mathrm{~F})$, numeric (\%N) and gravimetric (\%) methods of each dietary item

\begin{tabular}{|c|c|c|c|c|c|c|c|c|c|c|c|c|}
\hline & \multicolumn{3}{|c|}{ Zona $1(n=18)$} & \multicolumn{3}{|c|}{ Zona $2(n=7)$} & \multicolumn{3}{|c|}{ Zona $3(n=13)$} & \multicolumn{3}{|c|}{ Zona $4(n=11)$} \\
\hline & F\% & $\mathrm{N} \%$ & $\mathbf{P} \%$ & F\% & N\% & $\mathbf{P} \%$ & F\% & N\% & $\mathbf{P} \%$ & F\% & N\% & $\mathbf{P} \%$ \\
\hline \multicolumn{13}{|l|}{ CEPHALOPODA } \\
\hline Dosidicus gigas & 94,44 & 80,20 & 98,73 & 100 & 64,04 & 99,06 & 100 & 81,38 & 99,01 & 90,91 & 40,98 & 72,15 \\
\hline Gonatus antarcticus & 5,56 & 0,50 & $<0,001$ & 28,57 & 7,02 & $<0,001$ & 7,69 & 1,38 & $<0,001$ & & & \\
\hline Histioteuthis sp. & 5,56 & 0,50 & $<0,001$ & 14,29 & 0,88 & $<0,001$ & 23,08 & 2,07 & 0,02 & & & \\
\hline Liocranchia reinhardti & & & & & & & 7,69 & 0,69 & $<0,001$ & & & \\
\hline Loligo gahi & & & & 28,57 & 7,90 & $<0,001$ & & & & & & \\
\hline Moroteuthis sp. & & & & 14,29 & 0,88 & 0,80 & 15,39 & 1,38 & $<0,001$ & & & \\
\hline Oegopsida indet. & & & & 71,43 & 6,14 & 0,001 & & & & & & \\
\hline Onychoteuthis banksii & 16,67 & 4,46 & 0,04 & 42,86 & 3,51 & 0,001 & 23,08 & 4,14 & 0,003 & 9,09 & 0,82 & $<0,001$ \\
\hline Sthenoteuthis oualaniensis & 33,33 & 5,94 & 0,02 & 28,57 & 4,39 & 0,004 & 30,77 & 3,45 & 0,01 & 9,09 & 0,82 & 0,001 \\
\hline Todarodes filippovae & & & & 42,86 & 3,51 & 0,003 & & & & 9,09 & 0,82 & $<0,001$ \\
\hline \multicolumn{13}{|l|}{ TELEOSTEI } \\
\hline Myctophidae & 22,22 & 4,56 & 0,06 & & & & 7,69 & 1,38 & 0,03 & & & \\
\hline Cubiceps pauciradiatus & & & & 14,29 & 1,75 & 0,129 & & & & & & \\
\hline Telesotei indet. & 5,56 & 0,50 & 0,03 & & & & & & & & & \\
\hline Talismania bifurcata & 5,56 & 0,50 & 0,38 & & & & & & & & & \\
\hline Teleostei 1 & 16,67 & 0,99 & 0,22 & & & & 23,08 & 2,07 & 0,36 & 36,36 & 3,28 & 4,62 \\
\hline Trachurus murphyi & 16,67 & 1,98 & 0,52 & & & & 15,39 & 2,07 & 0,58 & 45,46 & 53,28 & 23,22 \\
\hline Total & & 202 & 43479,9 & & 114 & 30090,7 & & 145 & 27886,4 & & 122 & 32782,7 \\
\hline
\end{tabular}


En la zona 1, de acuerdo a los descriptores cuantitativos, se pudo observar que tanto en frecuencia, número y peso, la presa más importante fue Dosidicus gigas $\mathrm{y}$, en un segundo nivel, Sthenoteuthis oualaniensis con una frecuencia de 33,3 \% (Tabla 1).

En la zona 2, Dosidicus gigas siguió siendo la presa más importante en frecuencia, número y peso, seguido en segundo nivel de importancia, por calamares oegópsidos no identificados, los cuales fueron importantes en frecuencia ( $F=71,4 \%$ ) (Tabla 1$)$.

En la zona 3, la presa con el porcentaje mas alto en frecuencia, número y peso fue Dosidicus gigas, y en segunda categoría de importancia a nivel de frecuencia fue Sthenoteuthis oualaniensis ( $\mathrm{F}=30,7$ \%) (Tabla 1).

En la zona 4, el ítem presa mas importante en frecuencia y peso fue Dosidicus gigas ( $\mathrm{F}=90,9 \%$; $\mathrm{G}=72,2 \%$ ), seguido por la presa Trachurus murphyi la cual fue más relevante en número ( $\mathrm{N}=53,3 \%)$ (Tabla 1).

La frecuencia de ocurrencia de las especies de presas no se asoció significativamente entre zonas de pesca $\left(\chi^{2}=43,16\right.$, g. $\left.1 .=45, P=0,55\right)$, debido principalmente a que $X$. gladius se alimenta preferentemente de $D$. gigas en todas las zonas de pesca.

La abundancia de presas entre las zonas de pesca no fueron distinta (ANOVA, F(3, 45) $=0,37 ; P=0,76$ ) y estuvieron entre el rango de 11 a 16 presas. Tampoco fueron distintas la riqueza (ANOVA, $F(3,45)=0,38$; $P=0,76)$, diversidad (ANOVA, F(3, 45) = 0,05; $P=0,98)$, equitabilidad (ANOVA, $\mathrm{F}(3,45)=1,23$; $P=0,30)$ y dominancia (ANOVA, F(3, 45) =0,38; $P=0,83$ ) entre zonas de pesca (Tabla 2 ).

Tabla 2

\section{Abundancia, riqueza, diversidad, equitabilidad y dominancia de las presas en los contenidos estomacales de $X$. gladius en cada zona de pesca}

Prey abundance, richness, diversity, evenness and dominance from stomach contents of $X$. gladius in each fishing zone

\begin{tabular}{lcrrrrrrr}
\hline & \multicolumn{2}{c}{ Zona 1 } & \multicolumn{2}{c}{ Zona 2} & \multicolumn{2}{c}{ Zona 3 } & \multicolumn{2}{c}{ Zona 4} \\
& Promedio & \multicolumn{1}{c}{ D.E. } & Promedio & \multicolumn{1}{c}{ D.E. } & Promedio & D.E. & Promedio & D.E. \\
\hline Abundancia & 11,28 & 10,95 & 16,29 & 19,35 & 11,15 & 9,62 & 11,09 & 8,84 \\
Riqueza & 2,22 & 1,22 & 2,71 & 2,63 & 2,46 & 1,66 & 2,00 & 0,63 \\
Diversidad & 0,32 & 0,32 & 0,37 & 0,57 & 0,31 & 0,32 & 0,35 & 0,24 \\
Equitabilidad & 0,45 & 0,38 & 0,26 & 0,34 & 0,37 & 0,35 & 0,57 & 0,34 \\
Dominancia & 0,81 & 0,21 & 0,86 & 0,22 & 0,84 & 0,17 & 0,79 & 0,16 \\
\hline
\end{tabular}

\section{Discusión}

Los resultados muestran que Xiphias gladius, en el periodo de otoño del año 2004, se alimentó sobre un espectro trófico constituido por 10 especies de cefalópodos y 5 especies de peces, marcando una clara preferencia alimenticia por los primeros. Estos resultados concuerdan con la dieta de esta especie descrita en Chile en donde se encontró que se alimentaba principalmente de especies de cefalópodos y secundariamente de peces (Ibáñez et al. 2004). La mayoría de los estudios de la alimentación de $X$. gladius, comparativamente, han reportado una mayor cantidad de especies presas (Scott \& Tibbo 1968: 31 especies; Stillwell \& Kholer 1985: 36 especies; Clarke et al. 1995: 42 especies; Hernández-García 1995: 38 especies; Markaida \& Sosa-Nishizaki 1998: 35 especies; Salman 2004: 34 especies; Markaida \& Hochberg 2005: 25 especies de cefalópodos), exceptuando algunos trabajos que han encontrado igual o menor número de especies presa (Toll \& Hess 1981: 15 especies de cefalópodos; Moreira 1990: 14 especies; Bello 1991: 11 especies de cefalópodos; Peristeraki \& Tserpes 2001: 11 especies; Ibáñez et al. 2004: 14 especies; Peristeraki et al. 2005: 9 especies de cefalópodos). El bajo número de especies presas ha podido deberse a los pequeños tamaños de muestra considerados en este estudio. Otra explicación es que 
debido a las altas abundancias de D. gigas en Chile, sobre todo en los últimos años (Ibáñez \& Cubillos, 2007), la dieta del pez espada se centre en estos calamares y sea casi monoespecífica.

Los descriptores cuantitativos utilizados ponen en evidencia la marcada tendencia del pez espada a un alto consumo de $D$. gigas en las distintas localidades de estudio, considerándola como la presa más importante para esta especie (Tabla 1), similar al año anterior, pero en una mayor escala espacial (Ibáñez et al. 2004). La alta frecuencia de aparición de cefalópodos, específicamente $D$. gigas, en los contenidos gástricos demuestra la preferencia de $X$. gladius por los calamares musculares que forman agregaciones en la superficie, principalmente ommastréfidos epipelágicos, y otros calamares mesopelágicos (Palko 1981, Bello 1991, Markaida \& Sosa-Nishizaki 1998, Ibáñez et al. 2004, Markaida \& Hochberg 2005). Además, D. gigas es la especie de calamar más abundante y de mayor tamaño corporal en el Pacífico oriental (Nigmatullin et al. 2001) y en Chile en los últimos años (Ibáñez \& Cubillos 2007).

La dieta, en las cuatro localidades de captura, no fue distinta en cuanto a la frecuencia de ocurrencia de las presas, como tampoco en abundancia, riqueza, diversidad, equitabilidad y dominancia. En todas las zonas de pesca la oferta ambiental podría haber sido similar a juzgar por la abundancia y diversidad de las especies de peces y cefalópodos encontrados en los contenidos gástricos, los cuales son comunes en las zonas de pesca en donde se capturaron los peces espada (Kong \& Meléndez 1991, Rocha 1997). Sin embargo, la falta de tendencia a la asíntota de las curvas de rarefacción indican que el esfuerzo de muestreo no fue suficiente para representar y describir adecuadamente la dieta de $X$. gladius, por lo cual no existe suficiente evidencia para rechazar la hipótesis nula (X. gladius no presenta diferencias en la dieta entre las zonas de pesca). Por lo tanto es muy importante para los estudios de alimentación de peces considerar el tamaño muestreal para poder describir y realizar comparaciones de la dieta. Se sugiere evaluar este aspecto antes de realizar el muestreo o los análisis, ya que un insuficiente tamaño muestreal podría llevar a conclusiones erróneas.

\section{Agradecimientos}

Los autores agradecen a la tripulación de las embarcaciones Moroni y Nefi, por facilitar la recolección de las muestras de estómagos como en el año anterior (ver Ibáñez et al. 2004). También agradecemos los valiosos comentarios de Unai Markaida en la preparación del manuscrito y a tres evaluadores anónimos.

\section{Literatura citada}

Acuña E, JC Villarroel \& $R$ Grau. 2002. Fauna íctica asociada a la pesquería de pez espada (Xiphias gladius Linnaeus). Gayana 66(2): 263-267.

Barbieri M, M Donoso, A González, V Correa, B Leiva, A Montiel \& E Yañez. 1998. Development and present state of the swordfish, Xiphias gladius, fishery in Chile. En: Barrett I, O Sosa-Nishizaki \& N Bartoo (eds). Biology and Fisheries of Swordfish, Xiphias gladius. NOAA Technical Report NMFS 142: 1-10.

Bello G. 1991. Role of cephalopods in the diet of the swordfish, Xiphias gladius, from the eastern Mediterranean sea. Bulletin of Marine Science 49(1-2): 312-324.

Carey FG \& BH Robison. 1981. Daily patterns in the activities of swordfish, Xiphias gladius, observed by acoustic telemetry. Fishery Bulletin 79: 277-292.

Clarke MR, DC Clarke, HR Martins \& HM Silva. 1995. The diet of swordfish (Xiphias gladius) in Azorean waters Arquipelago. Ciências Biológicas e Marinhas 13: 53-69.

Cortés E. 1997. A critical review of methods of studying fish feeding based on analysis of stomach contents: application to elasmobranch fishes. Canadian Journal of Fisheries and Aquatic Science 54: 726-738.

De Sylva DP. 1962. Red-water blooms off northern Chile, April-May 1956, with reference to the ecology of the swordfish and the striped marlin. Pacific Science 16: 271-279.

Falabella F, R Meléndez \& ML Vargas. 1995. Claves osteológicas para peces de Chile central, un enfoque arqueológico. Editorial Artegrama, Santiago, 208 pp. 
Hammer Ø, DAT Harper \& PD Ryan. 2001. PAST: Paleontological Statistics Software Package for Education and Data Analysis. Palaeontología Electrónica 4: 1-9.

Hernández-García V. 1995. The diet of the swordfish Xiphias gladius Linnaeus, 1758, in the central east Atlantic, with emphasis on the role of cephalopods. Fishery Bulletin 93: 403-411.

Hulbert SH. 1971. The nonconcept of species diversity: a critique and alternative parameters. Ecology 52: 577-585.

Hyslop J. 1980. Stomach contents analysis: Review of methods and their application. Journal of Fish Biology 17: 411-429.

Ibáñez CM, C González \& L Cubillos. 2004. Dieta del pez espada Xiphias gladius Linnaeus, 1758, en aguas oceánicas de Chile central en invierno de 2003. Investigaciones Marinas 32(2): 113-120.

Ibáñez CM \& LA Cubillos. 2007. Seasonal variation in the length structure and reproductive condition of jumbo squid Dosidicus gigas (Orbigny, 1835) off central-south Chile. Scientia Marina 71(1): 123-128.

Kong I \& $\mathbf{R}$ Meléndez. 1991. Estudio taxonómico y sistemático de la ictiofauna de aguas profundas capturada entre Arica e isla Mocha. Estudios Oceanológicos 10: 181.

LaMonte F \& DE Marcy. 1941. Swordfish, sailfish, marlin and spearfish. Ichthyology Contributions. International Game Fish Association, New York 1(2): 1-24.

Leible M \& $\mathbf{P}$ Miranda. 1989. El otolito sagitta en el reconocimiento de diferentes especies de teleósteos de la costa central de Chile. Boletín de la Sociedad de Biología de Concepción 60: 149-160.

Ludwig JA \& JF Reynolds. 1988. Statistical ecology, 337 pp. John Wiley \& Sons, New York.

Magurran AE. 1988. Ecological diversity and its measurement, 175 pp. Princeton University Press, Princeton, New Jersey.

Markaida U \& O Sosa-Nishizaki. 1998. Food and feeding habits of swordfish, Xiphias gladius L., off western Baja California. En: Barrett I, O Sosa-Nishizaki \& N Bartoo (eds). Biology and Fisheries of Swordfish, Xiphias gladius. NOAA Technical Report. NMFS 142: 245-259.

Markaida U \& FG Hochberg. 2005. Cephalopods in the diet of swordfish (Xiphias gladius) caught off the west coast of Baja California, Mexico. Pacific Science 59: 2541.
Moreira F. 1990. Food of the swordfish, Xiphias gladius Linnaeus, 1758, off the Portuguese coast. Journal of Fish Biology 36: 623-624.

Nesis KN. 1987. Cephalopods of the World. THF Publications. New Jersey. 351 pp.

Nigmatullin ChM, KN Nesis \& AI Arkhipkin. 2001. A review of the biology of the jumbo squid Dosidicus gigas (Cephalopoda: Ommastrephidae). Fisheries Research 54: 9-19.

Palko BJ, GL Beardsley \& WJ Richards. 1981. Synopsis of the biology of the swordfish, Xiphias gladius Linnaeus. FAO Fishery Synopsis 127: 1-21.

Peristeraki P \& G Tserpes. 2001. Feeding habits of swordfish (Xiphias gladius L., 1758) in the S. Aegean Sea. Proceedings of the $10^{\text {th }}$ Hellenic Congress of Ichthyologists 2001, pp. 25-28.

Peristeraki P, G Tserpes \& E Lefkaditou. 2005. What cephalopod remains from Xiphias gladius stomachs can imply about predator-prey interactions in the Mediterranean Sea? Journal of Fish Biology 67: 549-554.

Rocha F. 1997. Cephalopods in Chilean waters, a review. Malacological Review 30: 101-113.

Salman A. 2004. The role of cephalopods in the diet of swordfish (Xiphias gladius Linnaeus, 1758) in the Aegean Sea (Eastern Mediterranean). Bulletin of Marine Science 74: 21-29.

Sanders HL. 1968. Marine benthic diversity: a comparative study. American Naturalist 102: 243-282.

Scott WB \& SN Tibbo. 1968. Food and feeding habits of swordfish, Xiphias gladius, in the western north Atlantic. Journal of the Fisheries Research Board of Canada 25(5): 903-919.

Smale MJ. 1996. Cephalopods as preys. IV. Fishes. Philosophical Transactions of the Royal Society of London B 351: 1067-1081.

Stillwell CE \& NE Kohler. 1985. Food and feeding ecology of the swordfish Xiphias gladius in the western North Atlantic Ocean with estimates of daily ration. Marine Ecology Progress Series 22: 239-247.

Takahashi M, H Okamura, K Yokawa \& M Okazaki. 2003. Swimming behaviour and migration of a swordfish recorded by an archival tag. Marine and Freshwater Research 54(4): 527-534. 
Toll RB \& SC Hess. 1981. Cephalopods in the diet of the swordfish, Xiphias gladius from the Florida straits. Fishery Bulletin 79(4): 795-774.

Wolff G. 1982. A beak key for eight eastern tropical Pacific cephalopod species with relationships between their beak dimensions and size. Fishery Bulletin 26(2): 357-370.

Yáñez E, C Silva, MA Barbieri \& K Nieto. 1996. Pesquería artesanal de pez espada y temperatura superficial del mar registrada con satélites NOAA en Chile central. Investigaciones Marinas 24: 131-144.
Yatsu A. 1995. Zoogeography of the epipelagic fishes in the south Pacific Ocean sector of the subantarctic, with special reference to the ecological role of slender tuna Allothunnus fallai. Bulletin of the National Research Institute of Far Seas Fisheries 32: 1-145.

Zar JH. 1984. Biostatistical analysis, 718 pp. Prentice-Hall, New York.

Recibido el 28 de diciembre de 2006 y aceptado el 19 de marzo de 2007 